\title{
Ultrafast optical signal processing using semiconductor quantum dot amplifiers
}

\author{
Berg, Tommy Winther; Mørk, Jesper
}

Published in:

2002 IEEE/LEOS Annual Meeting Conference Proceedings

Link to article, DOI:

10.1109/LEOS.2002.1134059

Publication date:

2002

Document Version

Publisher's PDF, also known as Version of record

Link back to DTU Orbit

Citation (APA):

Berg, T. W., \& Mørk, J. (2002). Ultrafast optical signal processing using semiconductor quantum dot amplifiers. In 2002 IEEE/LEOS Annual Meeting Conference Proceedings (Vol. 1, pp. 321-322). IEEE. https://doi.org/10.1109/LEOS.2002.1134059

\section{General rights}

Copyright and moral rights for the publications made accessible in the public portal are retained by the authors and/or other copyright owners and it is a condition of accessing publications that users recognise and abide by the legal requirements associated with these rights.

- Users may download and print one copy of any publication from the public portal for the purpose of private study or research.

- You may not further distribute the material or use it for any profit-making activity or commercial gain

- You may freely distribute the URL identifying the publication in the public portal

If you believe that this document breaches copyright please contact us providing details, and we will remove access to the work immediately and investigate your claim. 


\title{
Ultrafast Optical Signal Processing using Semiconductor Quantum Dot Amplifiers
}

\author{
Tommy W. Berg and Jesper Mørk \\ Research Center COM, Technical University of Denmark, Build. 345V, DK-2800 Kgs. Lyngby, Denmark \\ Phone: +454525 6379, Fax: +454593 6581, E-mail: twb@com.dtu.dk
}

\begin{abstract}
The linear and nonlinear properties of quantum dot amplifiers are discussed on the basis of an extensive theoretical model. These devices show great potential for linear amplification as well as ultrafast signal processing.
\end{abstract}

The rapid improvement in recent years within growth of Quantum Dot (QD) material has led to the availability of high quality gain material, which so far has been exploited mainly for QD lasers [1,2]. However, the unique properties of QDs offer many interesting features, which can be exploited in semiconductor optical amplifiers (SOAs) to be used either as linear amplifiers or as highly nonlinear elements. Among these features is a very broad gain spectrum, which is illustrated in fig. 1(a). The spectrum shows the presence of a fully inverted, inhomogeneously broadened, QD Ground State (GS) transition, an Excited State (ES) transition and a partly filled Wetting Layer (WL) in the shape of a narrow Quantum Well (QW). The width of the QD GS gain peak is significantly wider than that of a typical QW/bulk SOA, hinting to the potential of QD SOAs in WDM transmission systems.

Another unique aspect of QD SOAs is the low pumping required to obtain population inversion of the lower states in the dots and the resulting sharp saturation of the QD GS gain, illustrated in fig. 1(b). Such behavior has been observed experimentally [3] and owes mainly to the large energy separation between the QD states. A high inversion is crucial for both the linear and nonlinear properties of a QD amplifier as will be described in the following.

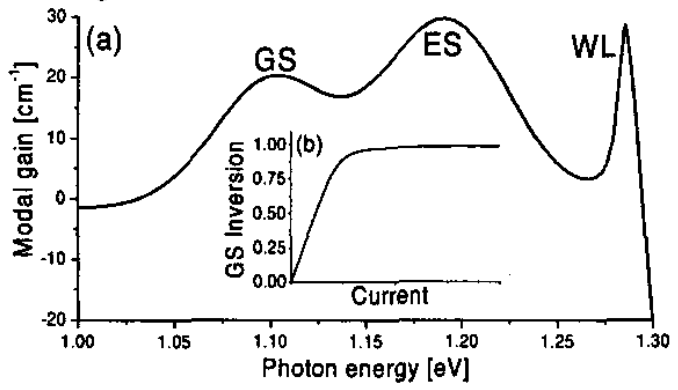

Fig 1: (a) Calculated gain spectrum of a typical $Q D$ device and (b) GS inversion as function of current.

Due to the low modal gain of QD SOAs it is necessary to use long devices in order to achieve a significant small signal gain. Amplified Spontaneous Emission (ASE) is known to limit the maximum achievable gain in long QW/bulk devices [4] and an interesting question is how ASE influences long QD SOAs. Fig. 2(a) shows the calculated small signal GS gain as function of device length [5]. The gain is seen to saturate at a high value of $47 \mathrm{~dB}$ for devices longer than $6 \mathrm{~mm}$, due to ASE. The explanation for this slow ASE saturation is a high saturation power (dashed line) of more than $20 \mathrm{dBm}$, which in turn owes to the high inversion of the active states, i.e. a low differential gain. The low modal gain, resulting in a low local stimulated recombination rate, combined with a relatively fast carrier relaxation into the active states means that capture and relaxation effects do not limit the performance in the regime considered here. As predicted earlier [6], the high inversion also results in a low noise figure at the GS transition (shown in fig. 2(b)) for devices shorter than the saturation length. However, as the ASE saturates the amplifier, the inversion of the GS decreases, resulting in a rapid increase of the noise figure.

The calculated saturation power and small signal gain are significantly higher than what is observed in QW/bulk devices indicating that QD SOAs are good candidates for high quality linear amplifiers.

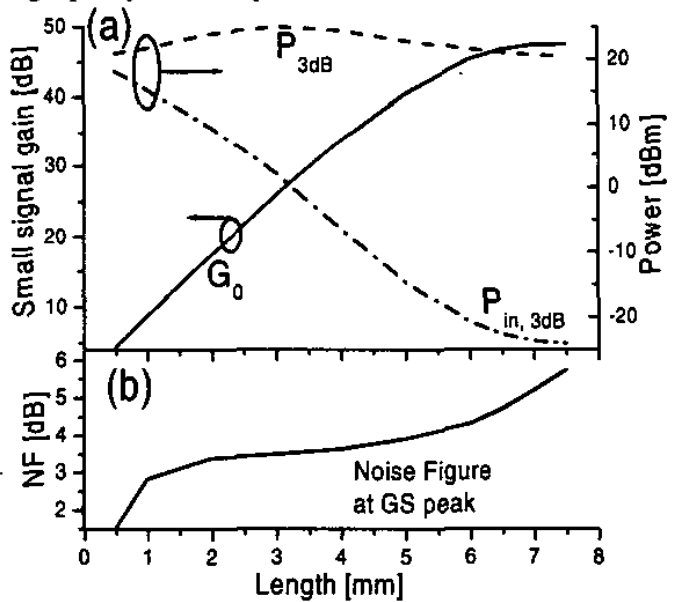

Fig. 2: (a) Small signal gain and saturation in- and output powers versus amplifier length. (b) GS noise figure.

The nonlinear properties of $Q D$ SOAs have been investigated by pump-probe measurements $[3,7]$. One of the experimental results (from [3]) are shown in fig. 3(a) (solid dots). An ultrafast gain recovery on a timescale of less than $150 \mathrm{fs}$ is observed after excitation with a short, $150 \mathrm{fs}$, pulse. The apparent lack of any slow component in the recovery is unique compared to other types of SOAs and hints to the possibility of using QD SOAs for ultrafast signal processing. Modeling results are shown in fig. 3(a) (solid line) and fig. 3(b). The later figure shows the dynamics of the GS, ES, and WL carrier populations during the recovery process. The GS is seen to recover by intradot relaxation of carriers from the, initially, completely filled 
ES, which in turn recovers on a longer timescale of picoseconds by capture of carriers from the WL. The ultrafast saturation and recovery of the GS gain thus corresponds to Spectral Hole Burning (SHB) and recovery in bulk/QW devices. The WL recovers on a ns timescale (shown in the insert of fig. 3(b)) determined by the carrier lifetime of the WL. The slow recovery of the WL indicates that under amplification of a train of pulses, with repetition period comparable to or shorter than the WL recovery time, the inversion of the structure will continue to decrease, thus slowing down the recovery of the GS, leading to strong patterning effects. The dynamics of the WL are thus important for the overall performance of the device. The possibility of employing optical holding or pumping in the WL has been investigated theoretically [9], indicating the potential for decreasing the recovery time of the WL significantly with this method. The decrease in the ES population after the amplification of a strong pump pulse, predicted by the modeling, has been confirmed experimentally [7].
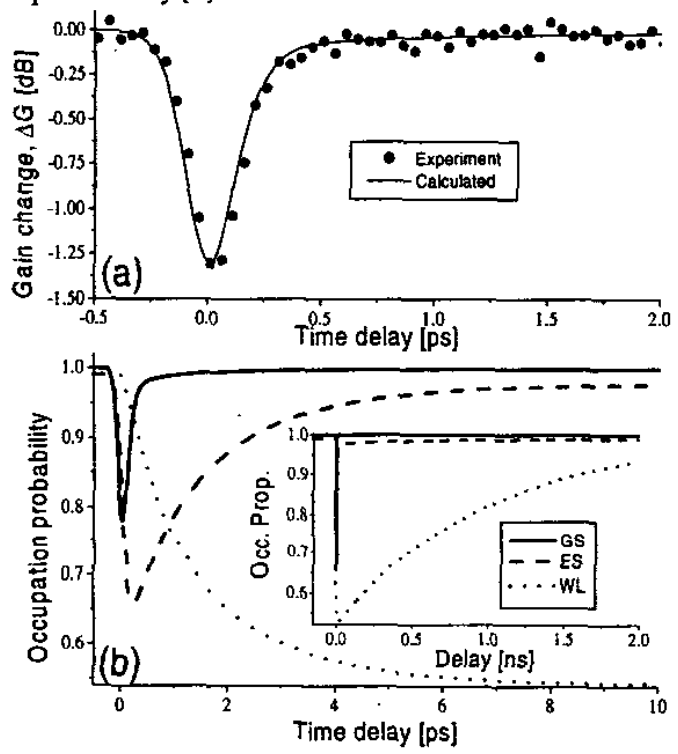

Fig. 3: (a) Measured (dots) [3] and calculated (solid line) gain recovery and (b) corresponding dynamics of the GS, $E S$, and WL populations.

The SHB effect may, however, be exploited under certain operating conditions. This can be illustrated by considering a simplified system consisting of a single $Q D$ level and a WL in the shape of a narrow QW. Fig. 4(a) shows the calculated small signal optical modulation efficiency of the gain versus average optical power when a weakly modulated signal is injected into the device. Considering first a device with fast carrier capture of $100 \mathrm{fs}$, corresponding to a typical dephasing time of a bulk/QW device, two distinct regimes are observed: low power levels, where the modulation efficiency is low but independent of average power, i.e. no pattern dependence; and high power levels, where the modulation efficiency is high but strongly dependent on the average power. It is in this latter regime that a typical device employing cross gain modulation, e.g. for wavelength conversion, would be operated. In the first regime the modulation is dominated by SHB and in the second regime it is dominated by Carrier Density Pulsation (CDP). Considering now a device with a slower capture of $4 \mathrm{ps}$, corresponding to typical capture times of QD devices, we see that the SHB part of the modulation is increased significantly and we thus have a regime with high modulation efficiency and no pattern dependence. Operating the device in this latter regime and injecting a $100 \mathrm{Gbit} / \mathrm{s}$ signal together with a weak $\mathrm{CW}$ beam, the potential for ultrafast signal processing can be illustrated. The modulated output $\mathrm{CW}$ signal is shown in fig. 4(b), where a very efficient modulation with only slight pattern effect is observed. This regime of operation, which has also been investigated by Sugawara et al. [10], is not feasible in bulk/QW devices due the fast intraband scattering times of $50-100 \mathrm{fs}$.
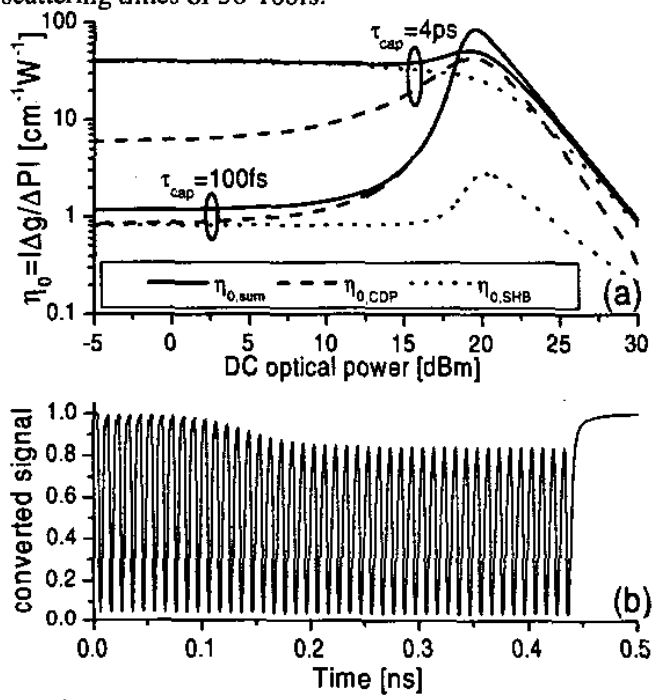

Fig. 4: (a) Optical modulation efficiency of a $Q D$ amplifier versus average power for two different values of the capture time, $\tau_{\text {cap }}$ (b) Normalized output signal from a $Q D$ wavelength converter when a worst-case signal is injected.

The field of QD SOAs still remains largely unexplored, but there seems to be great potential for improving the linear and nonlinear properties beyond those of bulk and QW devices.

[1] D. Bimberg et al., IEEE J. Select. Topics Quantum Electron. 3, p. 196 (1997)

[2] F. Klopf et al., IEEE Photon. Technol. Lett. 13, p. 764 (2001)

[3] P. Borri et al., IEEE J. Photon. Technol. Lett. 12(6), p. 594 $(2000)$

[4] T. Liu et al., Electron. Lett. 33(24), p. 2042 (1997)

[5] T.W. Berg, J. Mørk, OAA 2002, paper OWC5

[6] K. Komori et al., IEEE J. Photon. Technol Lett. 3(1), p. 39 (1991)

[7] K. Kim, T.B. Norris, P. Bhattacharya, LEOS 2001, paper WA2 [8] T.W. Berg et al., IEEE J. Photon. Technol Lett. 13(6), p. 541, (2001)

[9] T.W. Berg, J. Mørk, 28th ECOC 1, paper MoB2.4 (2001)

[10] M. Sugawara et al., Jpn. J. Appl. Phys. 40, p. L488 (2001) 